\section{OP0011 INTEGRATION OF FLOW AND DIGITAL CYTOMETRY IN EARLY TREATMENT-NAÏVE RHEUMATOID ARTHRITIS IDENTIFIES DISTINCT IMMUNOPHENOTYPES IN PERIPHERAL BLOOD AND DISEASE TISSUE}

F. Rivellese ${ }^{1,2}$, E. Pontarini ${ }^{1}$, L. Fossati-Jimack ${ }^{1}$, R. A. Moura ${ }^{3,4}$, V. C Romão $0^{3,4}$, J. E. Fonseca ${ }^{3,4}$, A. Nerviani ${ }^{1,2}$, C. Cubuk ${ }^{1}$, K. Goldmann $^{1}$, M. Bombardieri ${ }^{1,2}$, M. Lewis ${ }^{1,2}$, C. Pitzalis ${ }^{1,2}$ on behalf of Pathobiology of Early Arthritis Cohort (PEAC) investigators. ${ }^{1}$ William Harvey Research Institute, Centre for Experimental Medicine and Rheumatology, London, United Kingdom; ${ }^{2}$ Barts Health NHS Trust, Department of Rheumatology, London, United Kingdom; ${ }^{3}$ Hospital de Santa Maria, Centro Hospitalar Universitário Lisboa Norte, Lisbon Academic Medical Centre, Department of Rheumatology, Lisbon, Portugal; ${ }^{4}$ Instituto de Medicina Molecular, Faculdade de Medicina, Rheumatology Research Unit, Lisbon, Portugal

Background: The study of synovial tissue in patients with Rheumatoid Arthritis (RA) has led to the identification of synovial patterns of immune cell infiltration and specific cellular subsets associated that have been disease activity and clinical outcomes (1-3). However, the relationship of circulating and synovial immune cell sub-sets with histopathological features and clinical outcomes remains to be defined.

Objectives: To assess the relationship of peripheral blood and synovial immune cells with RA histopathology and clinical outcomes, by performing flow and digital cytometry in matched peripheral blood and synovial samples from patients with early RA.

Methods: 70 patients with early ( $<12$ months) untreated RA (2010 criteria) recruited in the pathobiology of early Arthritis Cohort (PEAC) at the Barts Health NHS Trust were included(1). Peripheral blood mononuclear cells $(n=70)$ were analysed by flow cytometry. Matched synovial tissues $(n=70)$ obtained by minimally invasive ultrasound-guided synovial biopsy underwent semi-quantitative scoring (0-4) of immune cell infiltration and classification into lympho-myeloid (LM), diffuse-myeloid (DM) and pauci-immune ( $\mathrm{PI}$ ) pathotypes, as previously described(1). 49 synovial and 36 matched peripheral blood samples underwent RNA-sequencing and were analysed by digital cytometry (Xcell) (4) and Singular Value Decomposition (SVD)

Results: Circulating B cells and their subsets showed significant inverse correlations with inflammatory markers (ESR, CRP), disease activity (swollen joints, four components and two components(5) DAS28) and ultrasound scores (Fig 1A). Among $T$ cell subsets, CXCR5-PD1hilCOS+CD4+ $T$ cells ( $T$ peripheral helper cells, Tph) had strong positive correlations with inflammatory markers (ESR and CRP), disease activity (DAS28) and ultrasound scores (Fig 1B). Tph in the peripheral blood also correlated with immune cell infiltration in synovia (Fig 1C) and were significantly higher in patients with a LM pathotype (Fig 1D). Accordingly, circulating Tph were associated with synovial LM pathotype independently of clinical features such as DAS28, ACPA positivity, Body Mass Index (BMI) and age (AUC 0.821). By applying digital cytometry in matched synovial and peripheral blood samples, synovial $B$ and T cells were significantly higher in patients with a LM pathotype, in line with the histological definition of the LM pathotype

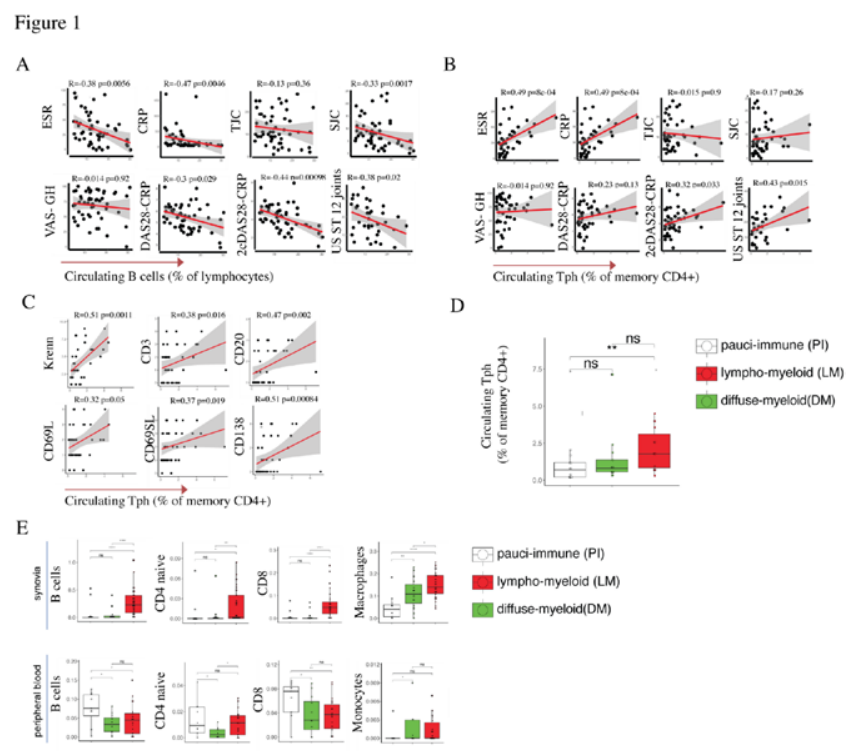

Figure 1.
- rich in B and T cells. On the contrary, circulating B cells and total CD4 and CD8 $T$ cells were significantly lower in patients with a synovial LM pathotype (Fig 1E). The Tph signature in synovia derived by Singular Value Decomposition (SVD) correlated with baseline ESR (R 0.38, $p<0.0001)$ and DAS28 (R 0.35, $p<0.0001)$ and with delta-DAS28 after 6 months of treatment with conventional synthetic DMARDs ( $\mathrm{R} 0.27, \mathrm{p}$ 0.026). Finally, the baseline synovial Tph signature was significantly higher in patients who progressed to the use of biologics and was predictive of future biologic DMARDs use, independently of baseline DAS28, ACPA positivity, BMI and age (AUC 0.703).

Conclusion: By combining conventional flow cytometry in the peripheral blood and digital cytometry on matched synovial and peripheral blood samples, we highlight diverging associations of circulating immune cell subsets with synovial inflammation and pathotypes. Tph cells, in particular, emerge as predictors of lympho-myeloid synovial inflammation and disease progression.

\section{REFERENCES:}

[1] F. Humby et al., Ann. Rheum. Dis. 78, 761-772 (2019), doi:10.1136/ annrheumdis-2018-214539.

[2] M. J. Lewis et al., Cell Rep. 28, 2455-2470.e5 (2019), doi:10.1016/j. celrep.2019.07.091.

[3] D. a Rao et al., Nat. Publ. Gr. 542, 110-114 (2017), doi:10.1038/nature20810.

[4] D. Aran et al., Genome Biol. 18, 220 (2017), doi:10.1186/s13059-017-1349-1.

[5] E. M. A. Hensor et al., Rheumatology. 58, 1400-1409 (2019), doi:10.1093/ rheumatology/kez049.

Acknowledgements: The Pathobiology of Early Arthritis Cohort (PEAC) was supported by the MRC (grant 36661). Versus Arthritis provided funding infrastructure support (grant 20022). F. Rivellese is funded by an NIHR Transitional Research Fellowship (TRF-2018-11-ST2-002). We would like to thank the patients and the clinical and laboratory team (core team) at Queen Mary University of London.

Disclosure of Interests: None declared

DOI: 10.1136/annrheumdis-2021-eular.438

\section{\begin{tabular}{|l|l}
\hline OP0012 & ASSOCIATION BETWEEN PASSIVE SMOKING IN
\end{tabular} CHILDHOOD AND ADULTHOOD, AND RHEUMATOID ARTHRITIS: RESULTS FROM THE FRENCH E3N-EPIC COHORT STUDY}

${\text { Y. } \text { Nguyen }^{1,2} \text {, C. Salliot }}^{2,3}$, X. Mariette ${ }^{4,5}$, M. C. Boutron-Ruault ${ }^{2}$, R. Seror ${ }^{4,5}$ ${ }^{1}$ AP-HP.Nord - Hôpital Beaujon, Department of Internal Medicine, Clichy, France; ${ }^{2}$ Centre for Research in Epidemiology and Population Health (CESP), Université Paris Sud, INSERM U1018, Villejuif, France; ${ }^{3}$ Centre Hospitalier Régional D'Orléans, Department of Rheumatology, Orléans, France; ${ }^{4} A P$ HP Hôpital Bicêtre, Department of Rheumatology and National Reference Center for Rare Systemic Autoimmune Diseases, Le Kremlin-Bic être, France;

${ }^{5}$ Université Paris Sud - Faculty of Medicine, INSERM UMR1184, Le KremlinBicêtre, France

Background: Rheumatoid arthritis (RA) is a systemic autoimmune disease of multifactorial aetiology, which preferentially affects women. To date, active smoking has been the most reproducibly reported risk factor for anti-citrullinated protein antibodies (ACPA) positive RA, particularly persons who carry the HLADRB1-shared epitope (SE) alleles.

Objectives: We aimed to investigate the relationships between passive smoking in childhood (PSc) or in adulthood (PSa), and the risk of incident RA in a large prospective cohort of healthy French women.

Methods: The E3N-EPIC (Etude Epidémiologique auprès des femmes de la Mutuelle générale de l'Education Nationale) is a French prospective cohor study that investigates environmental factors associated with chronic diseases. It follows 98,995 healthy French women since 1990 covered by a national health insurance primarily involving teachers. RA cases have been previously identified with specific questionnaires and medication reimbursement database. Women were considered exposed to PSc if they self-declared staying in a smoky room several hours a day during childhood, and to PSa if they self-declared being exposed at least one hour a day to passive smoking while adults. We used Cox multivariable regression models with age as the timescale (model 1), adjusted on smoking status (never, current, or former smoker) and on the two types of passive smoking (model 2), and on educational level, and BMI (model 3). Stratified analyses were conducted depending on the active smoking status (never or ever-smoker).

Results: 79,806 women were included in the study. Mean $( \pm$ SD) age at cohort entry was $49.0( \pm 6.4)$ years. Among them, 698 incident RA cases were identified, diagnosed after a mean of $11.7( \pm 5.8)$ years after baseline. In the whole cohort, 10,810 (13.5\%) women were exposed to PSc, 42,807 (53.6\%) to PSa, $6,581(8.25 \%)$ were exposed to both, and $47,036(58.9 \%)$ were exposed to either In the whole population, PSc was positively associated with the risk of RA in all three models (HR 1.24; $95 \% \mathrm{Cl}$ [1.01 to 1.51] in Model 3). In stratified analyses 